\title{
BMJ Open Systematic review and meta-analysis of the effect of SGLT-2 inhibitors on microvascular outcomes in patients with type 2 diabetes: a review protocol
}

To cite: Dorsey-Treviño EG, Contreras-Garza BM, GonzálezGonzález JG, et al. Systematic review and meta-analysis of the effect of SGLT-2 inhibitors on microvascular outcomes in patients with type 2 diabetes: a review protocol. BMJ Open 2018;8:e020692. doi:10.1136/ bmjopen-2017-020692

- Prepublication history and additional material for this paper are available online. To view these files, please visit the journal online (http://dx.doi. org/10.1136/bmjopen-2017020692).

EGD-T and BMC-G contributed equally.

Received 20 November 2017 Revised 29 May 2018 Accepted 1 June 2018
Check for updates

For numbered affiliations see end of article.

Correspondence to Dr René Rodriguez-Gutierrez; rodriguezgutierrez.rene@mayo. edu

\section{ABSTRACT}

Introduction Sodium glucose cotransporter 2 (SGLT-2) inhibitors are a relatively new drug-class of glucoselowering medications. Several trials and systematic reviews have demonstrated their beneficial effect on some macrovascular outcomes. Their effect on microvascular outcomes has been reported as positive in several trials, however, their effect remains uncertain. Therefore, we report the protocol of a systematic review and metaanalysis aimed at determining the effect of SGLT-2 inhibitors regarding patient-important and surrogate microvascular outcomes in patients with type 2 diabetes. Methods and analysis A comprehensive search will be conducted to find eligible articles from each database's earliest inception to November 2017. These databases will include Ovid, MEDLINE, EMBASE, Web of Science, and Scopus. We will search for randomized controlled trials (RCTs) that compare any of the SGLT-2 inhibitors with any other active treatment or placebo assessing microvascular outcomes in either their primary or secondary outcomes. Reviewers working independently and in duplicate will review all abstracts, and full-text manuscripts for eligibility, and will systematically extract the data and will assess the risk of bias in the included studies. Random-effects models will also be used.

Ethics and dissemination The results of the systematic review will be disseminated via publication in a peerreviewed journal regardless of outcome and will be presented at relevant conferences. The data we will use do not include individual patient data, so ethical approval is not required

PROSPERO registration number CRD42017076460.

\section{INTRODUCTION}

Globally, diabetes represents the leading cause of end-stage renal disease/chronic renal disease, blindness, clinical peripheral neuropathy and amputations. ${ }^{12}$ Therefore, decreasing the risk of the aforementioned microvascular complications is a priority for any diabetes therapeutic intervention and
Strengths and limitations of this study

- One limitation of this systematic review is that data availability and heterogeneity of outcomes definitions may vary among studies.

- Another limitation is that outcomes from microvascular complications will be mostly from secondary outcomes.

- A third limitation of this systematic review is that patient-important outcomes are scarcely reported in $20 \%$ of trials; therefore, data may not be enough to draw precise conclusions.

- One strength of this review is that this will be the first systematic review and meta-analysis designed to specifically assess the body of evidence regarding the effectiveness of sodium glucose cotransporter 2 inhibitors in patients with type 2 diabetes regarding microvascular outcomes.

represents a major concern for any healthcare system. Different strategies involving a rigourous glycemic control have been adopted to reduce this risk. Nonetheless, the paradigm of tight glycemic control (ie, independently of the drug used) will result in a decreased risk of microvascular complications has recently been dispelled. Recently, a systematic review reported no effect of tight glycaemic control (haemoglobin A1c (HbA1c) $<7.0 \%$ ) when compared to conventional glycaemic control (HbA1c $8.0-8.5 \%$ ) regarding patient-important microvascular outcomes (eg, end-stage renal disease, blindness, clinical neuropathy) in patients with type 2 diabetes. ${ }^{3}$ Still, there is a positive, however, inconsistent effect regarding surrogate markers (eg, microalbuminuria, photocoagulation). ${ }^{3}$ Other strategies, such as lipid lowering (eg, fibrates), antiplatelet agents, 
smoking cessation, blood pressure control including ACE inhibitors and life-style modification, in most cases, as a multifactorial intervention have been reported to have a positive effect, however, mostly over surrogate markers.

To date, there are at least 10 classes of antihyperglycaemic medications with different mechanism of action, efficacy, adverse events, costs and convenience. ${ }^{45}$ Sodium glucose cotransporter 2 (SGLT-2) inhibitors are one of the novel and most used class of antihyperglycaemic drugs and as a group are positioning themselves as a promising therapeutic class in current diabetes treatment. ${ }^{6-8}$ Previous systematic reviews have demonstrated their effectiveness in reducing HbAlc, body weight, blood pressure ${ }^{9-11}$ and the risk of major cardiovascular outcomes, including mortality. ${ }^{12}$ Their effect on microvascular patient-important outcomes and intermediate (ie, surrogate) markers has been reported in several individual trials to be positive; however, across some of the major randomised controlled trials (RCTs) their effect appears to be inconsistent and imprecise, and remains in great extent uncertain. ${ }^{13-19}$

Therefore, we plan to conduct a systematic review and meta-analysis to determine the effect of SGLT-2 inhibitors on patient-important and surrogate microvascular outcomes in adult patients with type 2 diabetes.

\section{METHODS AND ANALYSIS \\ Study design}

This protocol adheres to the Preferred Reporting Items for Systematic Reviews and Meta-Analyses Protocol (see online supplementary file).$^{20}$

\section{Eligibility criteria}

We will search for RCTs that compare any of the SGLT-2 inhibitors with any other active treatment or placebo, evaluating microvascular outcomes in either their primary or secondary outcomes. We will consider studies enrolling adults (18 years or older) with type 2 diabetes defined by any recognised standard diagnosis criteria, regardless of its evolution time, and with a minimum of $\geq 4$ weeks of intervention. We will exclude patients with any other diagnosis of diabetes (type 1 diabetes, maturity onset diabetes of the young (MODY), iatrogenic, gestational diabetes, drug-induced diabetes or any other disease that impairs secretion of insulin). There will be no language restriction, and we will exclude studies with missing data despite author contact.

\section{Study identification}

The search strategy will be designed by two experienced librarians (AMF and NA-V) with input from the study's principal investigators (NA-V and RR-G). A comprehensive search will be conducted to find eligible articles in several databases from each database's earliest inception to November 2017. These databases will include Ovid, MEDLINE, EMBASE, Web of Science and Scopus. Controlled vocabulary supplemented with keywords will be used to search for studies evaluating the effect of SGLT-2 inhibitors on diabetes microvascular complications. The design and conduction of the search strategy will be finished around November 2017. After we complete the data extraction phase, we will conduct a second search using the same criteria mentioned above to update any missing trial published during the months of data extraction. We will consult experts in the field and the references from each included trial to identify studies missed by our search strategy.

\section{Selection of studies}

Reviewers working independently and in duplicate will review all abstracts and selected full-text manuscripts for eligibility. Prior to formal abstract screening, a pilot, between reviewers, will be carried out to clarify any misunderstandings and ensure adequate comprehension. Two reviewers working independently and in duplicate will screen all titles and abstracts of the selected articles to assess eligibility. In this phase, we will be highly sensitive and therefore whenever reviewers disagree, the article will be included into the full-text phase. Disagreements at full-text screening will be resolved by consensus. Reasons for non-eligibility will be documented by the reviewers. Chance-adjusted inter-rater agreement for the title/abstract screening and the full text will be calculated using the Kappa statistics. ${ }^{21}$ Before and after both screening phases, the total number of included and excluded articles will be documented, including reasons for exclusion. Selection of studies will be carried out from January to February 2018.

\section{Outcomes of interest}

Microvascular outcomes (ie, nephropathy, retinopathy, peripheral neuropathy and peripheral vascular disease) will be assessed. Also, we will assess patient-important outcomes for any of the complications mentioned before. For this protocol, we defined microvascular complications and patient-important outcomes as:

\section{Nephropathy}

Surrogates: doubling of the serum creatinine, macroalbuminuria and microalbuminuria, and estimated glormerular filtration rate (eGFR). Patient-important outcomes: end-stage renal disease defined as a need for continuous renal replacement therapy or renal transplant, chronic renal disease stage $\geq$ III or renal death.

\section{Retinopathy}

Surrogates: changes from baseline of retinal neovascularisation, cataract extraction, event reported in general as retinopathy, retinal photocoagulation and treatment with intravitreal agents. Patient-important outcomes: diabetes-related blindness, vitreous haemorrhage, retinal detachment, severe macular oedema and retinal artery occlusion.

\section{Peripheral neuropathy}

Surrogates: changes from baseline of tendon reflexes, and electrophysiological parameters such as nerve 
conduction velocity and sensory conduction velocity. Patient-important outcomes: (pain, numbness), sensory loss (touch, vibration and quality of life).

\section{Peripheral vascular disease}

Surrogate: abnormal ankle-brachial index and/or arterial Doppler ultrasonography. Patient-important outcomes: prolonged wound healing, ulcers or amputation.

\section{Data collection process}

Two reviewers working independently will collect data for all the eligible articles. To standardise data extraction, a web-based data extraction form will be designed including information about type of study, baseline patient characteristics, drug being studied and effectiveness regarding microvascular complications. Two or more reviewers working independently and in duplicate will conduct a pilot phase to assess any disagreement; disagreements will be discussed and resolved by consensus. If any disagreement cannot be resolved by consensus, a third reviewer will arbitrate the final decision. If necessary, modifications on the form will be effectuated based on the feedback of the reviewers to get optimal calibration. Data collection will take place around March-April 2018.

\section{Missing data}

If major data (mean, median, SD, IQR, OR, effect sizes, number of participants, etc) regarding our primary or secondary outcomes is not clear, missing or presented in a form that is unextractable from the full text, an email will be sent to the corresponding author or the drug manufacturer to clarify the situation. After a lapse of 10 days, a second email will be sent to the non-responders. If the second attempt is unsuccessful, other authors will be contacted. If none of the authors or manufacturers respond, we will exclude the study. Every author and manufacturer contact will be documented.

\section{Risk of bias in individual studies and quality assessment}

Two review authors working independently and in duplicate will use the Cochrane risk of bias tool to assess the quality of RCTs based on the following domains: random sequence generation (selection bias), allocation concealment (selection bias), blinding (performance bias and detection bias), incomplete outcome data (attrition bias) and selective reporting (reporting bias). We will also evaluate the overall quality of evidence for each outcome using the Grading of Recommendations Assessment, Development, and Evaluation (GRADE) ${ }^{22}$ Disagreement will again be resolved by consensus or if not possible, by arbitration of a third reviewer. Risk of bias in individual studies will be assessed around March-April 2018.

\section{Sensitivity analysis}

To explain possible inconsistencies across study results, we will conduct the following subgroup analysis: patients with long-term versus recent diabetes diagnosis, patients with arterial hypertension as comorbidity versus patients without arterial hypertension, and trials of primary versus secondary cardiovascular prevention. If possible, we will also try to analyze different drug doses.

\section{Summary measures and data synthesis}

A narrative synthesis of the findings from the included studies will be provided, considered type of intervention, target population characteristics, type of outcome and intervention content. We will provide summaries of intervention effects for each study by calculating risk ratios (for dichotomous outcomes) or standardised mean differences (for continuous outcomes). When more than one study provide data on the same outcome measure, using the same type of intervention and comparator, a cumulative meta-analysis will be performed. Statistical analyses will be performed using Review Manager V.5.3. and results pooled following random-effects models in order to best address the heterogeneity in population characteristics across studies. $\mathrm{X}^{2}$ test and the $\mathrm{I}^{2}$ statistic will be used to assess heterogeneity between studies. A $\chi^{2}$ cut-off value of $\mathrm{p}<0.10$ and an $\mathrm{I}^{2}$ value $>50 \%$ will be considered as indicative of considerable heterogeneity not explained by chance. To explore causes of inconsistency and subgroup-treatment interactions, we developed protocol prespecified subgroup analyses (previously mentioned in the sensitivity analysis section).

\section{Patient and public involvement}

No patients or public were involved in the study.

\section{DISCUSSION}

We anticipate this review will provide highly relevant information for clinicians, policy-makers and guideline-makers that will benefit from a summary of the best available evidence regarding the effect, in patients with type 2 diabetes, of SGLT-2 inhibitors over microvascular complications to counsel their patients and make higher quality recommendations, accordingly. Also, this information might be important in those patients in whom a new glucose-lowering drug is needed. At this point, patients and clinicians will carefully have to consider the benefits aside glucose reduction in light of the potential risks of each drug class.

Several limitations can be foreseen of this review for instance, data availability and heterogeneity of outcome definitions may vary among studies. Probably, most of the included trials will have as primary objective glucose measures (ie, HbAlc) or macrovascular complications, hence, data for microvascular outcomes will stem mostly from secondary endpoints. Also, as patient-important outcomes are evaluated by less than $20 \%$ of trials, data of RCTs evaluating patient-important outcomes may not be enough to have precision regarding their effect. However, this will be the first systematic review and meta-analysis designed to specifically assess the body of evidence regarding the effect of SGLT-2 inhibitors in patients with type 2 diabetes regarding microvascular outcomes. In addition, the review will be based on an extensive and 
systematic literature search and will represent the best estimate of effect from the available body of evidence.

\section{Author affiliations}

${ }^{1}$ Endocrinology Division, Department of Internal Medicine, Hospital University "Dr. José E. González" Universidad Autónoma de Nuevo León, Monterrey, Mexico ${ }^{2}$ Plataforma INVEST Medicina UANL - KER Unit Mayo Clinic (KER Unit México), Universidad Autónoma de Nuevo León, Monterrey, México

${ }^{3}$ ResearchUnit, University Hospital “Dr. José E. González”, Universidad Autónoma de Nuevo León, Monterrey, México

${ }^{4}$ Knowledge andEvaluation Research Unit in Endocrinology, Mayo Clinic, Rochester, MN, USA

${ }^{5}$ Mayo Medical Library, Mayo Clinic, Rochester, MN, USA

${ }^{6}$ Division of Endocrinology,Diabetes, Metabolism and Nutrition, Department of Medicine, Mayo Clinic, Rochester, MN, USA

Contributors RR-G, EGD-T and BMC-G designed and wrote the protocol. EGD-T, AS-M, VG-N, GR-T, ADG-C, JGG-G, VMM and RR-G made substantial contribution and revision to it. NA-V and AS-M working with AMF will design the search strategy for this review. EGD-T, BMC-G, AS-M, VG-N, GR-T and ADG-C will undertake data collection. EGD-T and BMC-G will perform the statistical analysis of data. RR-G, EGD-T and BMC-G will interpret the results and write the final manuscript. JGG-G and VMM will work as second reviewer and third reviewer, respectively. The definitive version of this protocol reflects the contribution of all authors. All authors read and approved the final manuscript.

Funding This research received no specific grant from any funding agency in the public, commercial or not-for-profit sectors.

Competing interests None declared.

Patient consent Not required.

Provenance and peer review Not commissioned; externally peer reviewed.

Open access This is an open access article distributed in accordance with the Creative Commons Attribution Non Commercial (CC BY-NC 4.0) license, which permits others to distribute, remix, adapt, build upon this work non-commercially, and license their derivative works on different terms, provided the original work is properly cited and the use is non-commercial. See: http://creativecommons.org/ licenses/by-nc/4.0/

(c) Article author(s) (or their employer(s) unless otherwise stated in the text of the article) 2018. All rights reserved. No commercial use is permitted unless otherwise expressly granted.

\section{REFERENCES}

1. Singleton JR, Smith AG, Russell JW, et al. Microvascular complications of impaired glucose tolerance. Diabetes 2003:52:2867-73.

2. Centers for Disease Control Prevention. Diabetes report card 2012: centers for disease control and prevention, US Department of Health and Human Services. 2014. www.cdc.gov/diabetes/library/reports/ congress.html

3. Rodríguez-Gutiérrez R, Montori VM. Glycemic control for patients with type 2 diabetes mellitus: our evolving faith in the face of evidence. Circ Cardiovasc Qual Outcomes 2016;9:504-12.

4. Rodbard HW, Jellinger PS, Davidson JA, et al. Statement by an American Association of Clinical Endocrinologists/American
College of Endocrinology consensus panel on type 2 diabetes mellitus: an algorithm for glycemic control. Endocr Pract 2009;15:540-59.

5. Cheng AYY. Canadian Diabetes Association 2013 clinical practice guidelines for the prevention and management of diabetes in Canada.. Can J diabetes;2013:S1-3.

6. Inzucchi SE, Bergenstal RM, Buse JB, et al. Management of hyperglycemia in type 2 diabetes, 2015: a patient-centered approach: update to a position statement of the American Diabetes Association and the European Association for the study of diabetes. Diabetes Care 2015;38:140-9.

7. Harper W, Clement M, Goldenberg R, et al. Pharmacologic management of type 2 diabetes. Can J Diabetes 2016;40:193-5.

8. Qaseem A, Barry MJ, Humphrey LL, et al. Oral pharmacologic treatment of type 2 diabetes mellitus: a clinical practice guideline update from the american college of physicians. Ann Intern Med 2017;166:279-90.

9. Baker WL, Smyth LR, Riche DM, et al. Effects of sodium-glucose cotransporter 2 inhibitors on blood pressure: a systematic review and meta-analysis. J Am Soc Hypertens 2014;8:262-75.

10. Zhang Q, Dou J, Lu J. Combinational therapy with metformin and sodium-glucose cotransporter inhibitors in management of type 2 diabetes: systematic review and meta-analyses. Diabetes Res Clin Pract 2014;105:313-21.

11. Vasilakou D, Karagiannis T, Athanasiadou E, et al. Sodium-glucose cotransporter 2 inhibitors for type 2 diabetes: a systematic review and meta-analysis. Ann Intern Med 2013;159:262-74.

12. Zinman B, Wanner C, Lachin JM, et al. Empagliflozin, cardiovascular outcomes, and mortality in type 2 diabetes. N Engl J Med 2015;373:2117-28.

13. Heerspink HJ, Desai M, Jardine M, et al. Canagliflozin slows progression of renal function decline independently of glycemic effects. J Am Soc Nephrol 2017;28:368-75.

14. Kohan $\mathrm{DE}$, Fioretto $\mathrm{P}$, Johnsson $\mathrm{K}$, et al. The effect of dapagliflozin on renal function in patients with type 2 diabetes. $J$ Nephrol 2016;29:391-400.

15. Ott C, Jumar A, Striepe K, et al. A randomised study of the impact of the SGLT2 inhibitor dapagliflozin on microvascular and macrovascular circulation. Cardiovasc Diabetol 2017;16:26.

16. Cherney DZ, Perkins BA, Soleymanlou N, et al. Renal hemodynamic effect of sodium-glucose cotransporter 2 inhibition in patients with type 1 diabetes mellitus. Circulation 2014;129:587-97.

17. Cherney DZI, Zinman B, Inzucchi SE, et al. Effects of empagliflozin on the urinary albumin-to-creatinine ratio in patients with type 2 diabetes and established cardiovascular disease: an exploratory analysis from the EMPA-REG OUTCOME randomised, placebocontrolled trial. Lancet Diabetes Endocrinol 2017;5:610-21.

18. Cherney D, Lund SS, Perkins BA, et al. The effect of sodium glucose cotransporter 2 inhibition with empagliflozin on microalbuminuria and macroalbuminuria in patients with type 2 diabetes. Diabetologia 2016:59:1860-70.

19. Dziuba J, Alperin P, Racketa J, et al. Modeling effects of SGLT-2 inhibitor dapagliflozin treatment versus standard diabetes therapy on cardiovascular and microvascular outcomes. Diabetes Obes Metab 2014;16:628-35.

20. Moher $\mathrm{D}$, Shamseer $\mathrm{L}$, Clarke $\mathrm{M}$, et al. Preferred reporting items for systematic review and meta-analysis protocols (PRISMA-P) 2015 statement. Syst Rev 2015;4:1.

21. McGinn T, Wyer PC, Newman TB, Keitz S, Leipzig R, Guyatt G. Tips for learners of evidence-based medicine: 3 . Measures of observer variability (kappa statistic). Can Med Assoc J 2004;171:1369-73.

22. Murad MH, Montori VM, loannidis JPA, et al. How to read a systematic review and meta-analysis and apply the results to patient care. JAMA 2014;312:171. 